\title{
Virtual Calibration Method for Diesel Engine by Software in The Loop Techniques
}

\author{
M. C. Cameretti ${ }^{1}$, E. Landolfi ${ }^{2}$, T. Tesone ${ }^{2}$ and A. Caraceni ${ }^{2}$ \\ ${ }^{1}$ Department of Industrial Engineering, University of Naples Federico II, \\ 80125 Naples, Italy \\ *Email: mc.cameretti@unina.it \\ Phone: +390817683299; Fax: +390812394165 \\ ${ }^{2}$ NETCOM Group, Naples, Italy
}

\begin{abstract}
The calibration of the engine control unit is increased for the development of the whole automotive system. The aim is to calibrate the electronic engine control to match the decreasing emission requirements and increasing fuel economy demands. The reduction of the number of tests on vehicles represents one of the most important requirements for increasing efficiency of the engine calibration process. However, the definition of the design of experiment is not straightforward because the data is not known beforehand, so it is difficult to process and analyse this data to achieve a globally valid model. To reduce time effort and costs the virtual calibration can be a valid solution. This procedure is called software in the loop (SIL) calibration able to develop a process to systematically identify the optimal balance of engine performance, emissions and fuel economy. In this work, a virtual calibration methodology is presented by using a two-stage model to get minimum exhaust emissions of a diesel engine. The data used are from a GT-Power model of a 3L supercharged diesel engine. The model is able to calculate the engine emissions for different engine parameters (such as the start of injection, EGR fraction and rail pressure) and from optimisation process, new injection start maps that reduce pollutant emissions are created.
\end{abstract}

Keywords: Virtual calibration; diesel engine; emissions; model-based calibration; software in the loop (SIL).

\section{NOMENCLATURE}

$\begin{array}{llll}\text { BMEP } & \text { average effective pressure } & \text { SFC } & \text { specific fuel consumption } \\ \text { BTQ } & \text { brake torque } & \text { SI } & \text { spark ignition } \\ \text { BKW } & \text { engine power } & \text { SiL } & \text { software in the loop } \\ \text { EGR } & \text { EGR fraction } & \text { SOI } & \text { start of injection } \\ \text { DI } & \text { diesel ignition } & \text { x } & \text { test } \\ \text { DoE } & \text { design of experiments } & \Theta & \text { vector } \\ \text { ECU } & \text { electronic control unit } & y & \text { response } \\ \text { HiL } & \text { hardware in the loop } & & \end{array}$

\section{INTRODUCTION}

In the last years, in the automotive industry, there has been a continuous evolution in the research's field and experimentation. The respect for the environment, which results in 
more and more restrictive anti-pollution regulations has led to an increase in the planning and development costs for the engine. The impact on polluting emissions and on performance is clear; sometimes the results depend on the type and technological level of the engine used for the tests and/or on how the test has been conducted and on the conditions of the explored engine too. Due to the increase of conflicting objectives in terms of political, environmental and economic conditions, engine calibration has assumed an important role. The "calibration" of the functions for the electronic control unit (ECU) is a parameterization of the data maps for the optimum engine operation.

Engine mapping is the process of modelling engine behaviour as a function of adjustable engine parameters. Usually, a primary application of engine mapping is the calibration of electronic engine controllers used to optimise the fuel efficiency of the engine subject to legislative limits on the emission of exhaust gases.

Nowadays, calibration processes become more complex due to the high degrees of freedom for new generation engines: modern engine systems are equipped with complex technologies such as, for example, multiple injections, exhaust gas recirculation and aftertreatment systems.

At the present, engine calibration [1] is performed mainly on the road and on test benches, but recent progress on ECU virtualization has also made it possible to use the software in the loop (SIL) as a development tool. In order to reduce costs and time effort, the process of restructuring the engine from the engine test room is reflected in the desktop environment. The term virtual calibration [2] refers to the acquisition of data using simulation methods rather than measurement. Currently, there is no standard way to virtually engine calibrate, the parameters to be controlled are numerous and the targets can be essentially good performance and/or the reduction of polluting emissions. Therefore, several ways can be followed in order to optimise the engine.

In this context, the authors propose an innovative methodology based on the Software in the Loop (SIL) techniques to define the optimal control parameters. In the present study, a virtual calibration of some significant engine parameters, (engine speed, EGR fraction, start of injection, average effective pressure and the rail injection pressure), is made for different operating conditions by using engine mappings created with the twostage method in the Matlab toolbox, in order to reduce the emission of pollutants, in particular the $\mathrm{NO}_{\mathrm{X}}$ amount, without varying the engine performance. The engine considered is a turbocharged Diesel engine simulated by a 1-D code and an experiment plan through the design of experiment (DOE) [3] method is realised. Finally, an optimisation model was used and validated by numerous test cases.

\section{State of the Art}

In the last years, several authors are investigating new simulation techniques to calibrate engine parameters to obtain the best performance and/or low emissions in every operating condition. To this aim, optimisation models can be a useful tool. The authors Zaglauer and Knoll [4] had the main purpose of their activities to improve optimisation techniques. In fact, they used an evolutionary algorithm to solve complex problems in a reasonable time. Another aim was to develop, from the methodological point of view, a strategy based on specific parameters that, changing according to appropriate DoE plans, allows the strategic calibration of the injection advance, obtaining low levels of emissions and consumption. The number of test points should be as low as possible, but large enough to obtain the data to create a statistical model that faithfully reproduces the description of the engine behaviour as the factors change, that are the engine parameters. This statistical 
model is used to determine the optimal control of the engine parameters.

Some studies are present in the scientific literature for the analytical determination of important parameters influencing combustion phase in an engine and of consequence its performance and emissions [5,6]. Several tools in a graphic interface language have been implemented by using software that allows interaction with the various devices and the test room in order to make the tests efficient and reduce their number. A further advantage of a virtual calibration is the possibility of having perfectly repeatable load cycles because not influenced by ambient conditions unlike those obtained on a roller bench or on the road that is affected by some variability [7].

As known, the core of the control operations is included in an electronic control unit named ECU which controls the engine. Data-based modelling techniques can be an attractive alternative to physical models to increase efficiency in the modelling process and if in combination with automated measurement can help to significantly accelerate the calibration process. A fast simulation speed of the resulting models allows their implementation into real-time simulation environments, such as hardware-in-the-loop (HiL) systems [8]. The calibration of an electronic system like the one that runs a modern engine requires a thorough knowledge not only of the engine hardware itself in terms of sensors [9] and actuators, but also of the software in the control unit that, according to the calibration strategies adopted, allows the compliance with emission regulations and obtaining the desired performance in terms of power and torque supplied, as well as fuel consumption.

To reduce calibration time, many companies switched to a model-based calibration process combined with test bench improvements to automate the data collection process. Model-based calibration techniques were used to adapt the models to the data collected using a DOE in order to more adequately understand the design space [10-12]. It is about using the design of experiment, statistical modelling and calibration in order to implement tables or models on a control unit, one of the software used is the model-based calibration toolbox (MBC). In the powertrain calibration industry, different types of modelling are used that incorporate DOE and statistical learning techniques for both steady-state and dynamic maps. The preferred methodology is the Gaussian process modelling [13], which for $\mathrm{NO}_{\mathrm{x}}$ and soot of a diesel engine [14] have demonstrated the efficient application of new statistical learning algorithms based on Gaussian processes in building accurate global engine models.

Calibrations in most cases rely on lookup tables due to calculation limits. In the case of the model-based calibration toolbox (MBC), if the calibration model has satisfied all the error criteria, it is implemented in the calibration generation toolbox (CAGE). The CAGE Toolbox is used to produce optimal measurements of calibration data. Modelbased calibration techniques reduce the calibration time and improve the quality of the control systems [15]. In this way, it is possible to obtain significant time savings. The activity can thus be preceded by a planning phase consisting of designing the experiment, choosing and optimising the type of mode. This type of methodology has also been adopted for the use of alternative fuels too, such as bioethanol.

Following the parameterization with the DOE method, a specific engine calibration was defined for the bio-ethanol-based mixture. All this by acting on a few parameters of the alternative mixture and then analysing the measured trends through an analysis of Pareto - Anova [16]. Gokce and others describe the model-based calibration for engine mapping. This method allows the knowledge of engine emissions by varying the valves timing, the rotation speed and the torque. In this way, it is possible to trace the minimum emission, for example, of carbon monoxide. At the end of the calibration, the 
look-up table was filled up with optimum cam angle values to be used in an electronic control unit of a diesel engine [17].

Data-based models can incorporate intrinsic unknown non-linearities into sampled data, resulting in more precise models that generally do not require any prior physical knowledge. Moreover, the remarkable simulation speed of the resulting models allows its implementation for real-time simulation using hardware in the loop (HiL) techniques [18]. The strong need for a reduction of calibration costs and time leads to a reduction of the test rooms. A further step may be to transfer all components in a single simulation environment, this procedure is called the software in the loop calibration [19] [20]. The data are no longer acquired through measurements, but via simulations, i.e. virtual calibration [21]. The data are collected by using software such as GT-Power. Many authors investigate on the virtual engine calibration using 1-D models as GT-Power code.

Some authors [22] proposed two effective methodologies to overcome some critical issues concerning the base calibration of engine control parameters. Specifically, neural networks and 1-D CFD simulation were alternatively adopted to reliably calibrate specific ECU functions starting from a reduced number of experimental data. In [23] the adopted optimisation approach shows the capability to reproduce with good accuracy the experimentally identified calibration. Using the optimisation process it is possible to calibrate with strategies such as closing and opening of the intake valves rather than a purely throttle-based calibration. By these models, the engine behaviour in the early stages of the design process could be better understand and can assist in identifying data collection requirements prior to testing [24]. The times will be reduced if compared to those when there is a physical model [25]. This approach allows a more extended optimisation and analysis, performing all operations before the engine is working [26].

\section{VIRTUAL CALIBRATION}

As said before, a method of calibration is model-based calibration, which provides applications and design tools for complex systems. For optimal calibration, it uses statistical modelling and numerical optimisation. It is possible to define test plans, to adapt statistical models to the considered engine and to generate optimal calibrations and lookup tables for complex engines with high degrees of freedom that would require many tests with traditional methods. With the help of some software it is possible to develop a process to systematically identify the optimal balance of engine performance, emissions and fuel economy, and reuse statistical models for design control, for hardware-in-theloop (HIL) tests or for powertrain simulation. In the last years, a complex network has led to many control units. One of the methods capable of developing vehicle functions under these conditions is software in the loop (SIL). This method, which is the subject of this paper, offers the possibility of developing and testing a function in its context within a vehicle through a certain number of phases.

The aim of this study is to calibrate some engine parameters, such as the injection advance angle (SOI, start of injection), of a diesel engine for different operating conditions by using engine mappings created with the two-stage method in the Matlab toolbox in order to reduce the emission of pollutants, specifically carbon monoxide (CO), unburned hydrocarbons $(\mathrm{HC})$ and mainly oxides of nitrogen $\left(\mathrm{NO}_{\mathrm{x}}\right)$, without modifying the torque with respect to the reference values. This calibration is referred to the process of experiment design, statistical modelling and techniques and optimisation tools to define a methodology for the efficient production of high-quality calibrations for complex applications. The MATLAB tool allows extracting the calibrations directly from the 
models making the analysis possible with a limited amount of time. Physical models that can be useful for understanding the engine behaviour at the beginning of the design process and for identifying data collection requirements before the testing.

The model represents a statistical simulation of the physical model. The statistical model requires less time than a physical model, allowing to perform optimisation and analyses before the engine is available. In the first phase, a 1-D engine model is built by GT-Power software and is enhanced with phenomenological sub-models. By this code it was possible to elaborate an engine map with the help of the DOE methodology, to reduce the number of tests that should be performed. In the second part of the work a parametric model, called two-stage model, has been created so it has been possible to carry out the optimisation phase which has the main purpose of reducing the time of the tests and to improve the quality of the results, in terms of pollutant emissions, with a torque constraint to not affect engine performance. The specific fuel consumption is kept under control without representing a constraint in the cases examined.

\section{Engine Model}

The first step of the "software in the loop" calibration is the engine modelling as shown in Figure 1. The software used to recreate the behaviour of the engine is the onedimensional GT-Power code. Initially, engine characteristics are inserted such as number of strokes, number of cylinders, bore and valves (Table 1), engine type (SI o DI), and then specify the used combustion and heat transfer model.

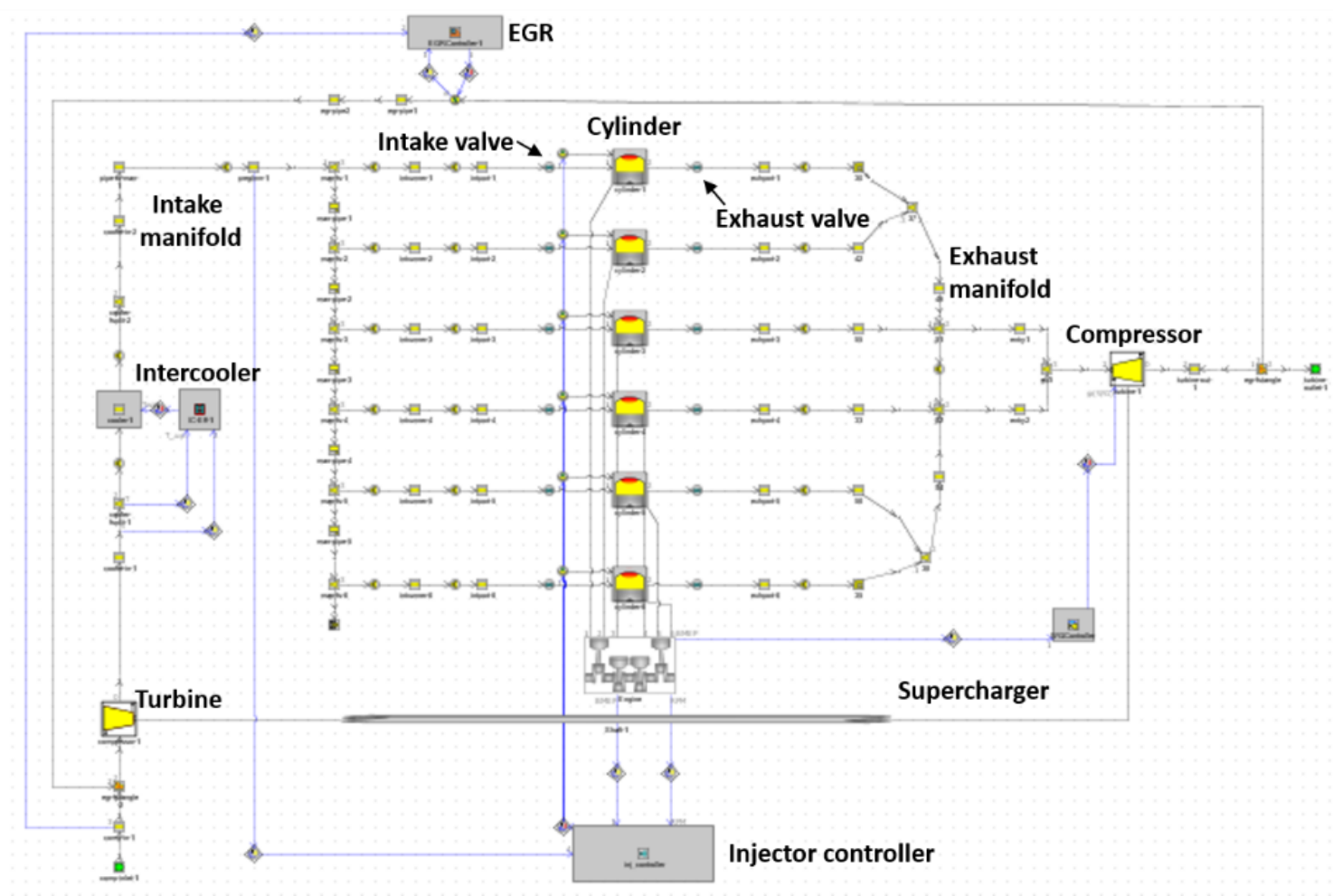

Figure 1. Engine GT-power model.

The chosen heat transfer model uses a simple correlation (1) to define the heat transfer between the hot gas and the cylinder walls. 
$Q=h_{c} A\left(T_{g}-T_{w}\right)+C \sigma\left(T_{g}^{A}-T_{w}^{A}\right)$

The first term represents the heat exchange by convection, and its intensity is given by defining the coefficient $h_{c}$ to the engine parameters:

$N u=\left(\frac{\hbar_{c} b}{k}\right)=a\left(\frac{\overline{S_{p}} b}{\mu}\right)^{d}$

Where $\mathrm{a}=0.35 / 0.8, \mathrm{~d}=0.7$. The second term calculates the irradiation, not negligible when the fuel is diesel oil. $\mathrm{C}=0.075, \sigma=$ Stefan-Boltzmann constant $0.57 \cdot 10-7 \mathrm{~W} /\left(\mathrm{m}^{2} \mathrm{~K}^{4}\right)$

In the cylinder, the combustion model is a Wiebe function. It models the combustion velocity in the diesel engines. The Wiebe function is used to simulate fuel burning. The Wiebe function for the mass fraction burned is given by:

$f=1-\exp \left[-a\left(\theta-\theta_{0} / \Delta \theta\right)^{n}\right]$

where $f=$ the fraction of heat added, $\theta=$ the instantaneous crank angle, $\theta_{0}=$ the start of combustion crank angle, $\Delta \Theta=$ the duration of the heat addition (combustion angle), $\mathrm{a}=5$ and; $\mathrm{n}=3$. The result can be calculated as the average value calculated in each cycle.

Table 1. Engine main characteristics.

\begin{tabular}{lc}
\hline Engine & 3.0 Multijet \\
\hline Type & 4 stroke \\
Number of cylinders & 6 \\
Valves/Cyl. & 2 \\
Bore $(\mathrm{mm})$ & 84 \\
Stroke $(\mathrm{mm})$ & 90 \\
Compression ratio & 16.5 \\
Torque $(\mathrm{Nm})$ & 550 \\
Power $(\mathrm{kW})$ & 177 \\
\hline
\end{tabular}

\section{Design of Experiment (DOE)}

As a second step, the design of the experiment has been made. The DOE is a set of statistical methodologies for modelling a system that is not yet known. Therefore, statistical methods have an important role in the planning, execution, analysis and interpretation of data. The traditional method of collecting large amounts of data, keeping each factor constant until all the possibilities have been tested, is an unfeasible approach due to the increase in the number of factors.

In the case of a discrete number of measured data, with the DOE methodology, it is possible to model a system whose behaviour is unknown. The DOE includes the creation of an experiment plan in accordance with statistical laws, to minimise measurement efforts, changing a variable at each measure. The model has created using the help of mathematical methods. It is important to note that each variable affects performance differently. Therefore, the aim of the planned experiment is to understand which set of variables in a process affect its performance. With the application of the DOE, it is possible to reduce drastically the costs of the experimental tests and, by correct 
planning, obtain results with a lot of useful information.

For the realisation of the DOE, it is necessary to introduce the characteristic parameters of the diesel engine model under investigation. In this case, the most significant factors for the specific problem are the engine speed, EGR fraction, start of injection (SOI), BMEP, average effective pressure and; $\mathrm{P}_{\text {rail,, }}$, injection pressure in the rail. For the rotation speed three levels have been chosen, $2500 \mathrm{rpm}, 3000 \mathrm{rpm}$ and $3500 \mathrm{rpm}$, while the EGR fraction was from 0.05 to 0.2 . The combination of the levels of the various factors generated a total of 900 experiments. Table 2 shows the selected engine parameters of the DOE and their interaction, as example. In this way, it is possible to create the reference engine map.

Table 2. DOE parameters.

\begin{tabular}{|c|c|c|c|c|c|c|c|}
\hline DOE Type: & \multicolumn{2}{|c|}{ Full Factorial } & $\checkmark$ & & & \# of Experiments: & 900 \\
\hline Factor & & & Min & $\operatorname{Max}$ & \# of Levels & & \\
\hline RPM & RPM & $\checkmark$ & 2500.0 & 3500.0 & 3 & & \\
\hline EGRfraction & fraction & $v$ & 0.05 & 0.2 & 5 & & \\
\hline startofinjection & deg & $\checkmark$ & -20.0 & 0.05 & 5 & & \\
\hline bmep & & & 12.0 & 18.0 & 4 & & \\
\hline injpres & bar & 2 & 1000.0 & 2000.0 & 3 & & \\
\hline
\end{tabular}

\section{Two-Stage Model}

The two-stage is a model designed in two phases for the engine calibration process. In this process, the data collection method consists in setting control parameters during each test, called "global", and changing a parameter, called "local", in a certain interval.

$y_{i}^{j}=f_{i}\left(x_{i}^{j}, \theta_{i}\right)+e_{i}^{j}$

Where subscript " $i$ " refers to the individual tests, " $j$ " to the different experiments in a test" $x_{i}{ }^{j}$ ", is the $j$-th value of the independent parameter, " $\theta_{i}$ " is the $\mathrm{i}$-th vector of the size parameters (rx 1 ), " $y_{i}{ }^{j}$ " is the $j$-th response of the $\mathrm{i}$-th test, " $e_{i}{ }^{j}$ " where it is a random error term that reflects the uncertainty in the response.

Figure 2 shows the structure of the two-stage model, specifically the relationship between local and global models. In this case, the input is the SOI and the global input is: pressure in the rail, EGR fraction, engine speed, BMEP effective average pressure. The desired responses are nitrogen oxide $\mathrm{NO}_{\mathrm{x}}$, carbon monoxide $\mathrm{CO}$, unburned hydrocarbons $\mathrm{HC}$, keeping the torque BTQ in a limited range with reference to the standard engine map. 


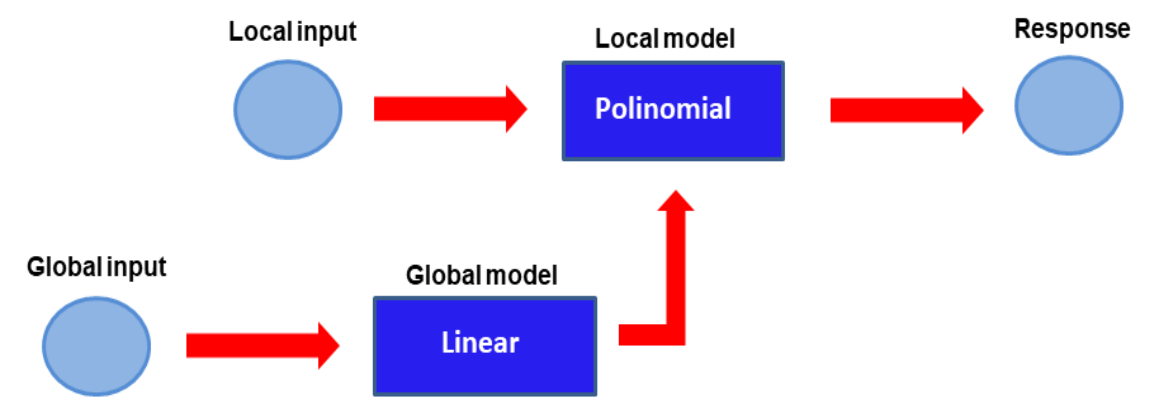

Figure 2. Two-stage model.

The creation of the Two-Stage model is carried out in a tool of Matlab for creating a statistical model of an automotive engine that can predict torque and pollutant emissions generated by the engine as a function of the angle of start of injection and/or other variables. It provides design tools to optimally calibrate complex powertrain systems. In the Model Based Calibration (MBC) the engine map will be imported. The aim is to produce optimal SOI, rail pressure and EGR fraction in order to reduce pollutant emissions, specifically $\mathrm{NO}_{\mathrm{x}}, \mathrm{CO}$ and $\mathrm{HC}$, without reducing the engine performance too much. The local model chosen in this work is the polynomial one, characterised by the following function:

$f\left(s o i^{2}\right) C O=333 * s o i^{2}+232 * s o i-46$

For global model the hybrid RBF model was chosen. This model is a forecasting technique that integrates regression trees, and ridge regression, with radial basis function (RBF). After an assessment of the residues, it is possible to obtain the results optimised. Figure 3(a) to 3(c) show the three-dimensional response surfaces related to the different estimation models of the pollutant exhaust emissions. These response surfaces represent the inputs used in CAGE tool used for the optimisation phase and described in the next section. From simulations many results have been carried out at variable load, only the high load results are reported.

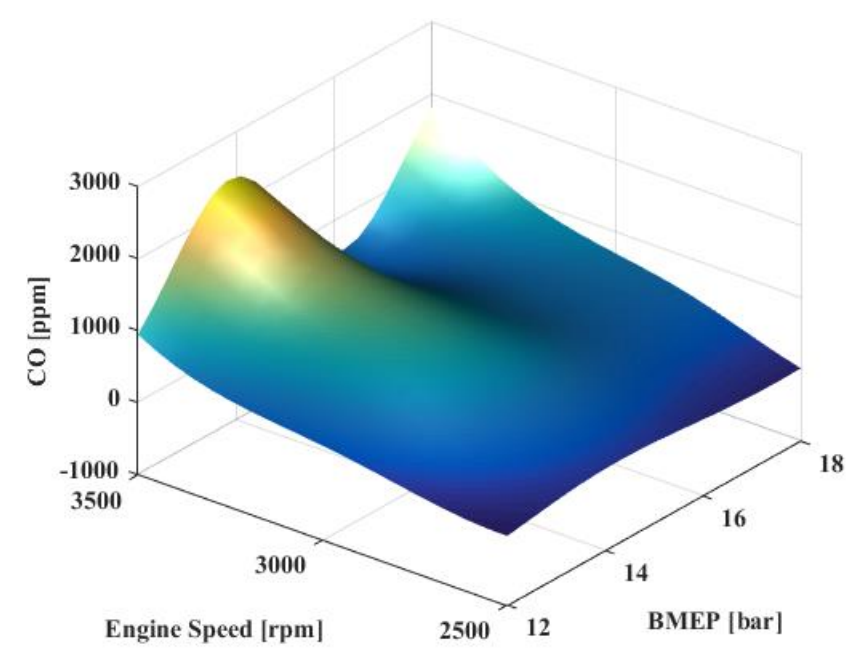

(a) 


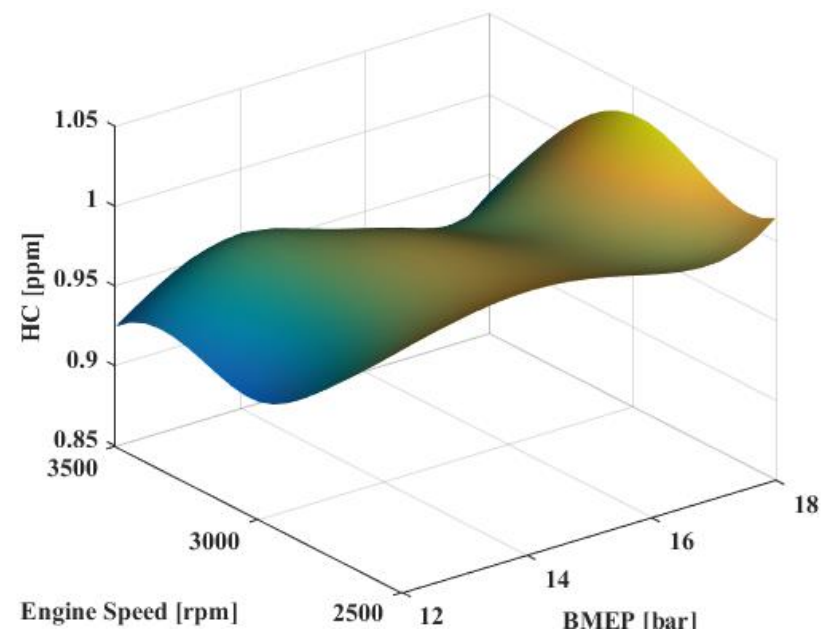

(b)

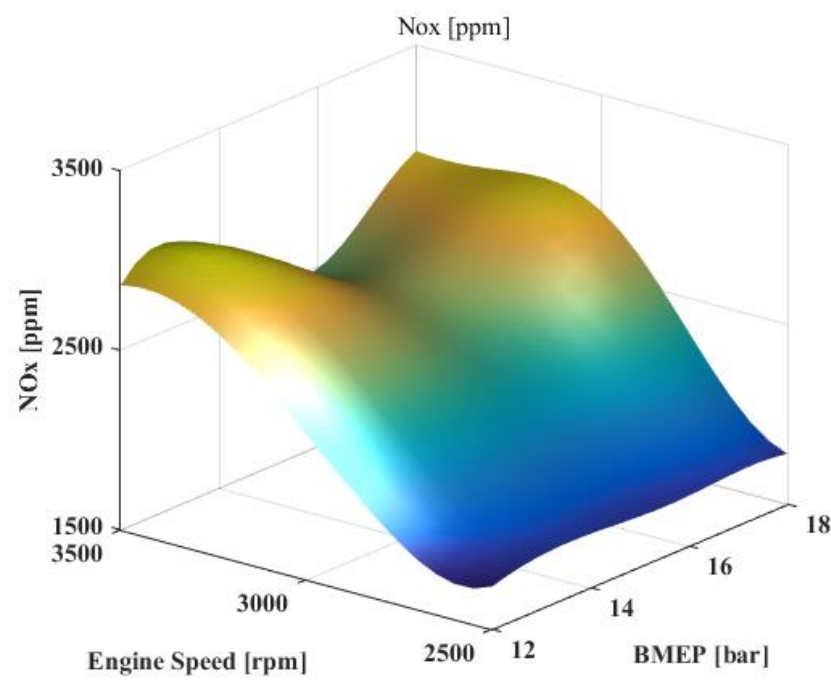

(c)

Figure 3. Three-dimensional response surface for (a) carbon monoxide, (b) unburned hydrocarbons and; (c) oxides of nitrogen.

\section{Description of Optimisation Procedure}

The optimisation process in Figure 4 provides the analytical models to calibrate the lookup tables generated previously. The main steps of the process are:

i. generation of optimal calibrations directly from empirical engine models;

ii. production of calibration tables (optimisation maps) by a tool named CAGE;

iii. comparison of calibrations with test data.

After generating the response surfaces, an optimisation was carried out for each pollutant. The calibration maps created are all characterized by the engine speed and the effective average pressure. The parameters to optimise are SOI, $\mathrm{P}_{\text {rail }}$, EGR fraction (recirculated exhaust mass/total mass) with the aim of reduce the polluting species considered. 


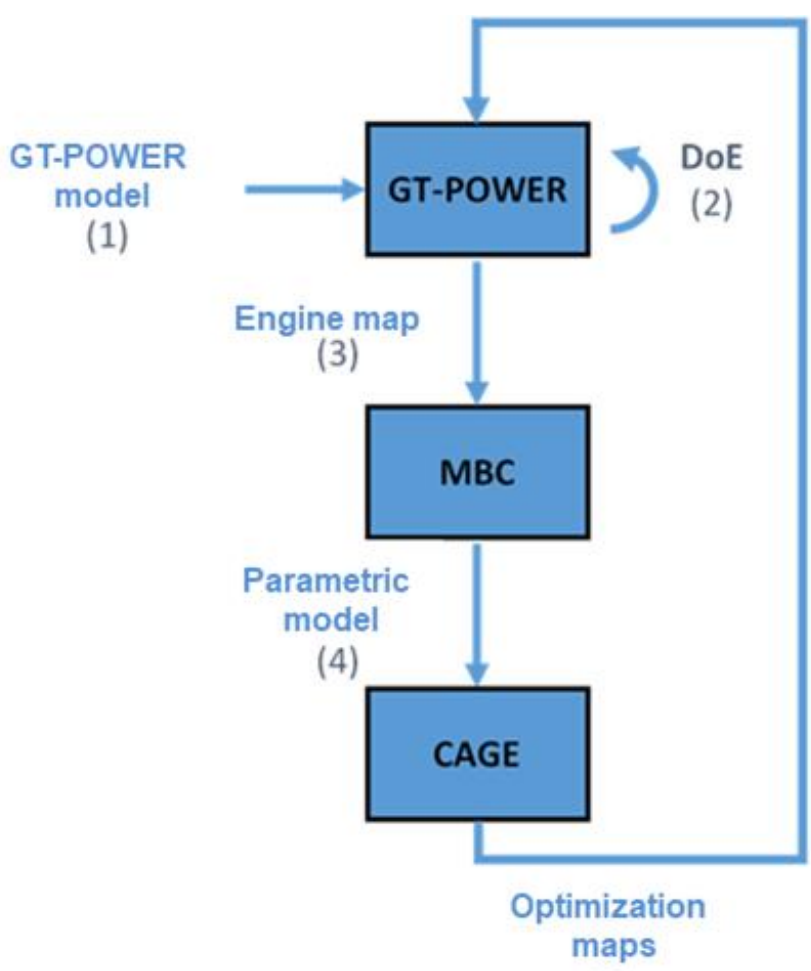

(5)

Figure 4. Optimisation procedure.

The constraints imposed refer to the values of the original engine map, because the fundamental objective of this work is to obtain an improvement in terms of emissions starting from the engine plan originally generated in GT-POWER in the first phase of this activity. Therefore, the new maps by varying the injection advance must provide lower polluting species preserving a torque value not too different respect to the original one. A right compromise between low polluting emissions and engine performance should be obtained. The result maps are shown in Figure 5(a) to 5(c). The optimised maps will be inserted into the engine model, in order to realise a new engine map with lower polluting emissions values than those of the original engine map.

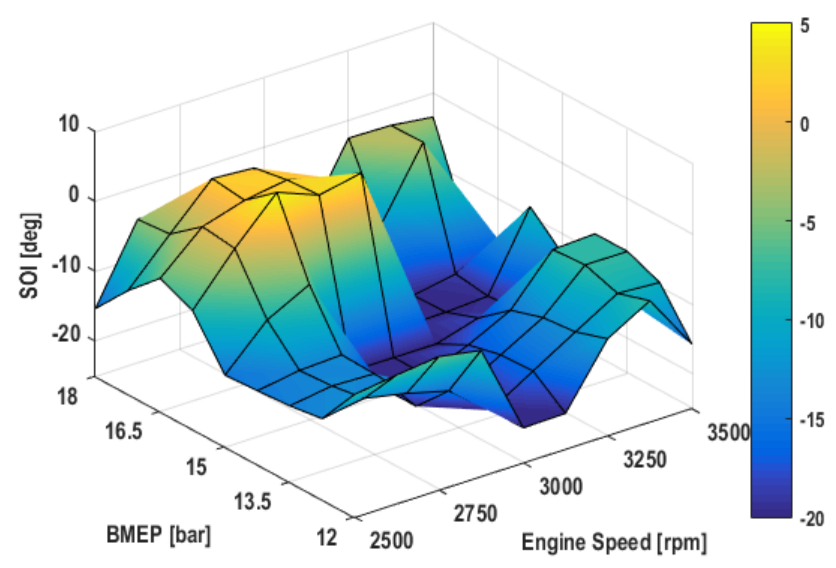

(a) 


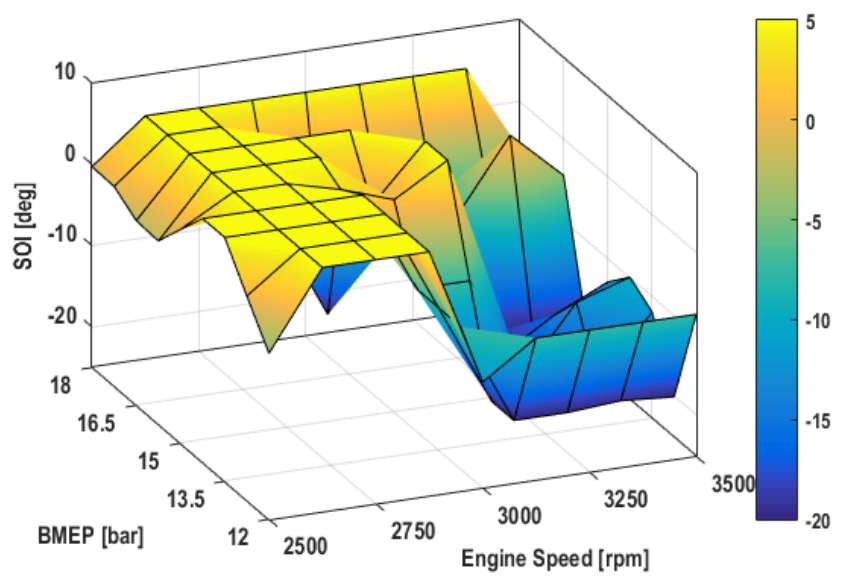

(b)

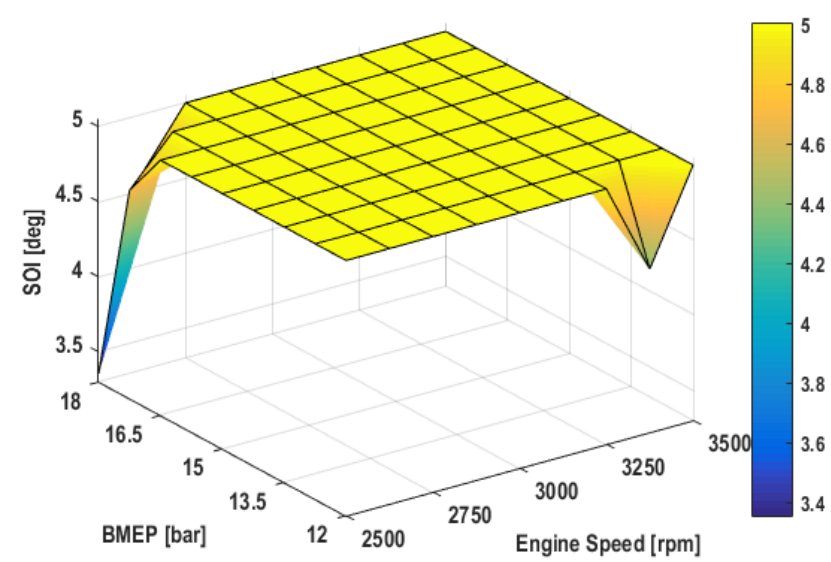

(c)

Figure 5. Map of the SOI optimised to (a) $\mathrm{CO}$, (b) $\mathrm{HC}$ and; (c) $\mathrm{NO}_{\mathrm{x}}$ reduction.

\section{RESULTS}

In this section, the results of virtual calibration process are analysed. The output of the CAGE software is the optimised maps with the start of injection, the rail pressure and the EGR fraction. In the last phase of the work, these optimised maps were imported into GTPower model and further simulations were carried out. These new simulations have led to real improvements. The created maps, shown in the previous paragraph, have been created simultaneously and their matching allowed to obtain the values optimised in various operating conditions. The focus was on reducing nitrogen oxides, a real critical issue in diesel engines. The reference parameter used was the injection advance.

In the previous section, the optimised map of the injection start angle has been created to reduce nitrogen oxides. Figure 6(a), 6(b) and 7 show the three variables considered (SOI, BMEP and EGR ratio) at various engine velocities. Figure 6(b) shows the optimised map of the rail pressure that reduces the $\mathrm{NO}_{\mathrm{x}}$ compared to those present in the original engine map. The pressure values are mainly below 1500 bar. In the representative Figure 7, the EGR fraction is about 0 . It therefore covers high values compared to those initially inserted. For higher EGR values a reduction of nitrogen oxides is attended. Since the EGR system has the purpose to the reduction of this polluting specie, the methodology used is validated. The optimised maps have led to improvement in terms of pollutants, validating the virtual calibration process. Nevertheless, it may 
happen that there are values of the nitrogen oxides higher than those of the original values.

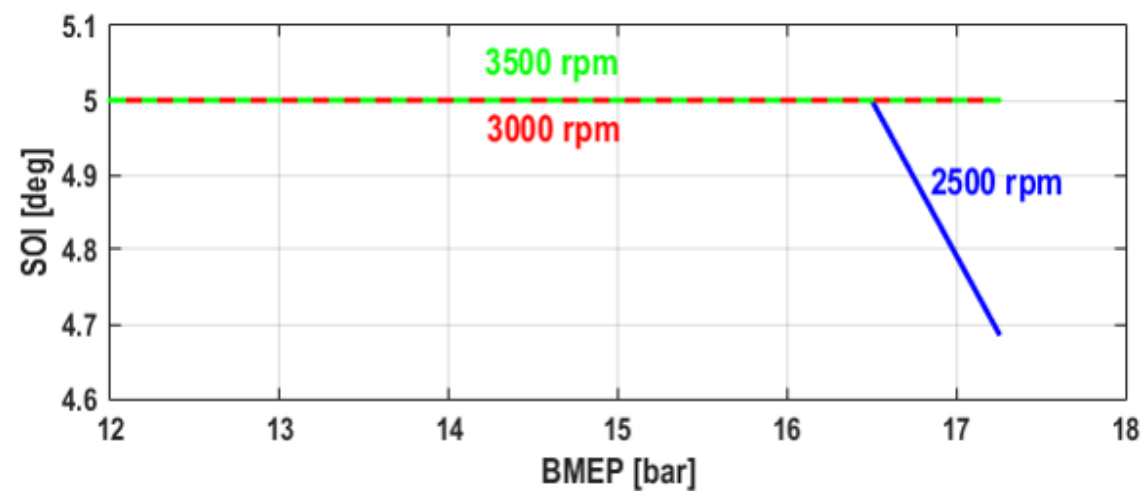

(a)

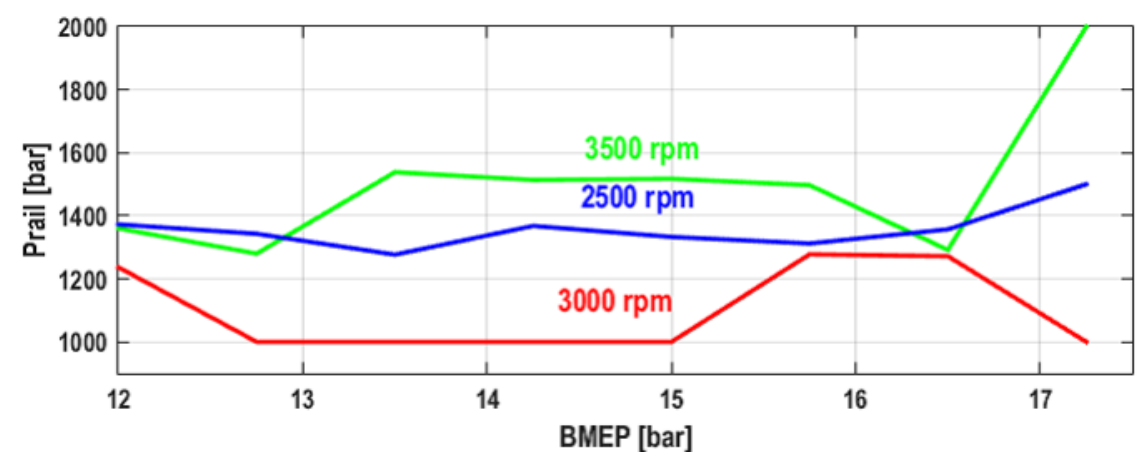

(b)

Figure 6. Optimised (a) SOI map and; (b) $\mathrm{P}_{\text {rail }}$ map to $\mathrm{NO}_{\mathrm{x}}$ reduction with isolines characteristic of the engine speed

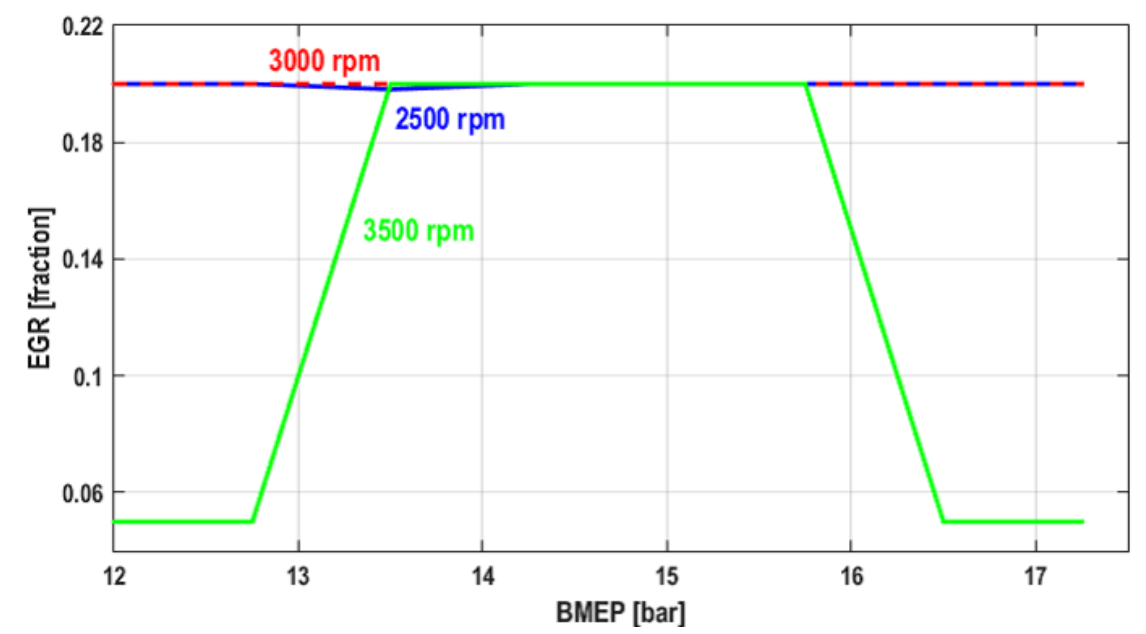

Figure 7. EGR optimised to $\mathrm{NO}_{\mathrm{x}}$ reduction at different engine speed.

In Figure 8(a) to 8(c) the improvement occurred after the optimisation in the case with EGR fraction equal to 0.05. In the images, the EGR ratio and the injection pressure for each test case are reported. The results obtained can be considered quite good, considering that the maps used in the model are the standard ones included in the software, no real data being available. The results show an evident improvement with the 
introduction of optimised maps. The optimised pressure values in the rail, start of injection and EGR fraction represent a compromise between the aim of reducing emissions and the desire to not penalize engine performance excessively. The number of data does not allow to report all the experiments carried out, for this reason, considering a pressure in the rail equal to 1000 bar and a fraction of EGR of $20 \%$, the Table 3 with the most significant trends observed is reported.

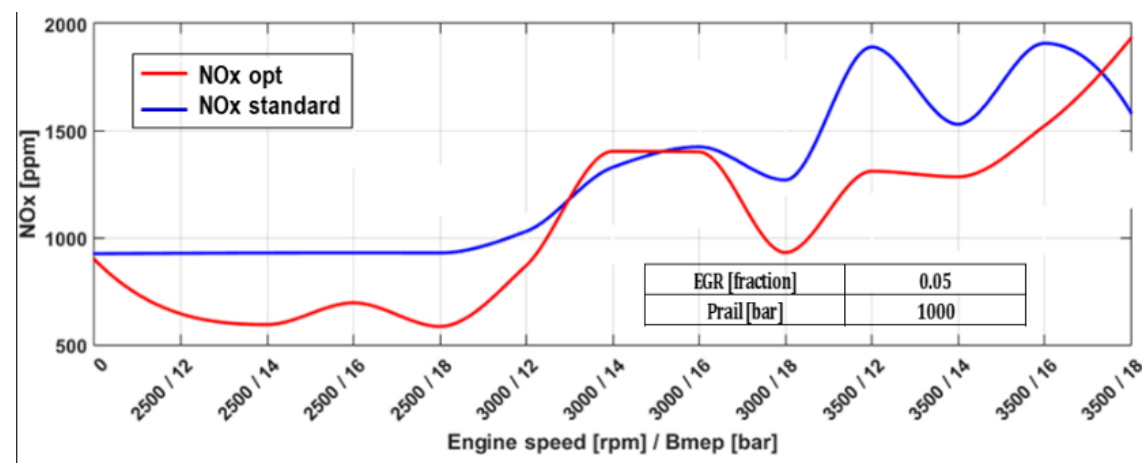

(a)

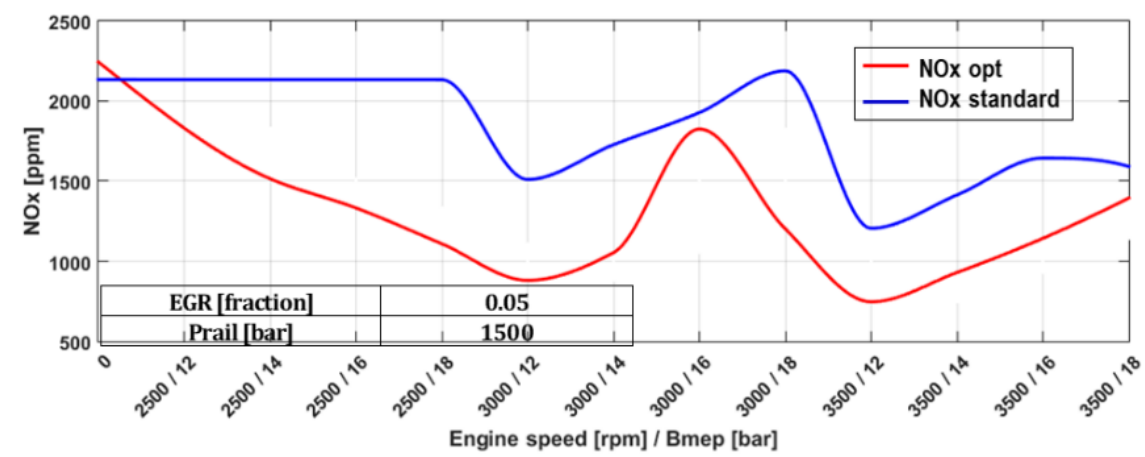

(b)

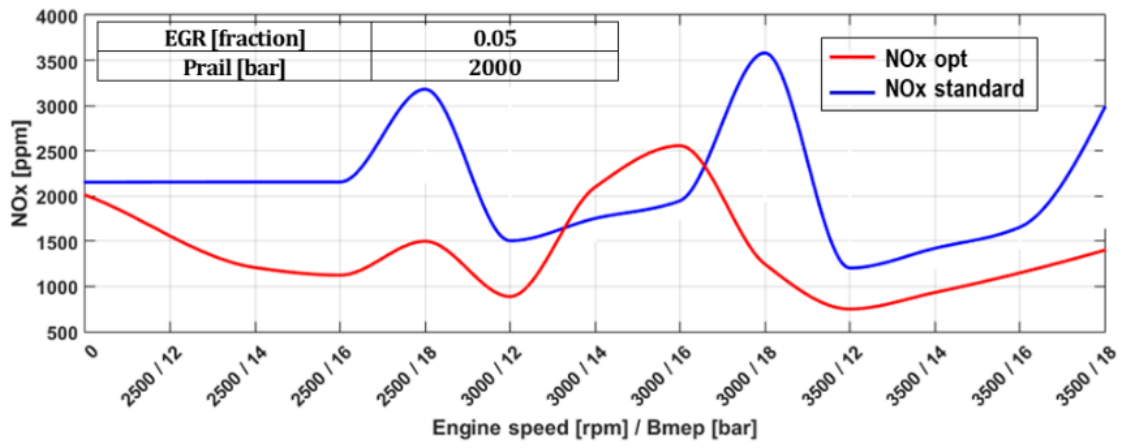

(c)

Figure 8. Nitrogen oxides comparison before and after (a) SOI optimisation and; (b), (c) optimisation.

From the results shown it is possible to find the trends of polluting emissions and engine performance for the test cases examined. In percentage terms, following optimisation of nitrogen oxides or optimisation of carbon monoxide, the mean results for all test cases are reported in Table 4. These are some obtained results with the optimisation, all have led to percentages of reduction of positive nitrogen oxides, which 
reach up to $30 \%$ in the case of EGR equal to $20 \%$. Furthermore, the reduction in performance does not have a significant value compared to the advantage obtained on the reduction of polluting emissions.

Table 3. Analysis of the results at $\mathrm{P}_{\text {rail }}=1000$ bar and $\mathrm{EGR}=20 \%$.

\begin{tabular}{lcccccccc}
\hline $\begin{array}{l}\text { Engine } \\
\text { speed } \\
(\mathrm{rpm})-\end{array}$ & $\begin{array}{c}\mathrm{NO}_{\mathrm{x}} \\
\mathrm{std} . \\
(\mathrm{ppm})\end{array}$ & $\begin{array}{c}\mathrm{NO}_{\mathrm{x}} \\
\mathrm{opt} \\
(\mathrm{ppm})\end{array}$ & $\begin{array}{c}\mathrm{BTQ} \\
\text { standard } \\
(\mathrm{Nm})\end{array}$ & $\begin{array}{c}\mathrm{BTQ} \\
\mathrm{opt} \\
(\mathrm{Nm})\end{array}$ & $\begin{array}{c}\mathrm{BKW} \\
\text { standard } \\
(\mathrm{kW})\end{array}$ & $\begin{array}{c}\mathrm{BKW} \\
\mathrm{opt} \\
(\mathrm{kW})\end{array}$ & $\begin{array}{c}\mathrm{m}_{\text {fuel }} \\
\text { standard } \\
(\mathrm{mg} / \mathrm{cycle})\end{array}$ & $\begin{array}{c}\mathrm{m}_{\text {fuel }} \\
\text { opt } \\
(\mathrm{mg} / \mathrm{cycle})\end{array}$ \\
\hline $2500-12$ & 926 & 643 & 309.5 & 294.7 & 81 & 77.2 & 47.815 & 40.615 \\
$2500-16$ & 928 & 695 & 310.1 & 287.3 & 81.2 & 75.2 & 48.198 & 51.528 \\
$3000-18$ & 1268 & 930 & 293.2 & 260.3 & 92.1 & 81.8 & 62.383 & 52.917 \\
$3500-14$ & 1529 & 1283 & 353.2 & 307.9 & 129.5 & 112.8 & 43.662 & 74.455 \\
\hline
\end{tabular}

Table 4. Results of optimisation of $\mathrm{NO}_{\mathrm{x}}$ and $\mathrm{CO}$.

\begin{tabular}{llcc}
\hline Optimisation of $\mathrm{NO}_{\mathrm{x}}$ & & \multicolumn{2}{c}{ Optimisation of CO } \\
\hline Variable & Reduction $(\%)$ & Variable & Reduction $(\%)$ \\
\hline $\mathrm{NO}_{\mathrm{x}}$ & $>30 \%$ & $\mathrm{CO}$ & $28 \%$ \\
Torque & $>10 \%$ & Torque & - \\
Power & $>10 \%$ & Power & - \\
$\mathrm{SFC}$ & $13 \%$ & $\mathrm{SFC}$ & $17 \%$ \\
\hline
\end{tabular}

\section{TOWARDS REAL-TIME CALIBRATION WITH HIL TECHNIQUES}

Finally, a brief mention was made, as a work in progress, on connecting the SIL techniques to real-time calibration by using HIL techniques. Starting from the GT-Power model it is possible to get three advantages at the same time:

i. define a virtual DoE;

ii. design a High-Fidelity model able to reliably simulate a real engine;

iii. design ECU models (soft-ECU) based on real information taken from ECU software, such as the calibration maps.

Therefore, an accurate tuning process is necessary, featured by comparing real data, obtained from fuel consumption maps from test cell, and virtual data, obtained by simulations. Nevertheless, the virtual calibration process up until now described is only executable in off-line mode; this is because it requires a considerable computational effort.

Consequently, it is not possible to directly export the high-fidelity model for realtime testing with hardware in the loop (HIL) [27] simulation. The hardware in the loop is a form of real-time simulation: a simulated system is connected to some real and simulated components present in the car. The components are sensors, actuators and the control unit, the object of the analysis. In this way, in a virtual environment, it is possible to verify the robustness of the algorithm by simulating different operating conditions, before they are installed on the vehicle. The repeatability of this process simplifies the identification of system defects and allows testing solutions to any problems and/or defects that arise.

Hence, the proposed methodology provides for a second step based on real-time testing, by using real ECU and an engine model for HIL applications. Generally, this kind 
of model is designed using Simulink software with real-time constrains, so it is computationally lighter. In particular, it is featured by two components:

i. Engine mean values model obtained converting some of the mechanical parts of High-Fidelity model (GT-Power) for Simulink real-time applications (i.e. cylinders block, air path, exhaust pipe);

ii. Parametric models obtained by fuel consumption map and/or experimental data, defining look-up-tables (i.e. starting conditions, some features of the gearbox, some transient condition).

Having the advantage of a high-fidelity model, that allows designing a virtual engine test cell, the proposed methodology allows adding new components to the realtime engine model, in order to accurately estimate emissions. In particular, these estimator models are obtained usually through neural-networks techniques oriented to machine learning methods.

The target of the second step of the proposed methodology is to design a fast realtime estimator starting from virtual or real engine data. For example, the data obtained from virtual DOE (off-line) are used as training-set (off-line) for the neural-networks model to execute on the real-time simulator (i.e. DSpace and/or National Instruments platforms). This implementation can use Simulink software and its tools for neuralnetworks, oriented to real-time execution, while estimators of the high-fidelity model could not have been executed in this way. With accurate real-time estimators, it is possible to test previously obtained virtual calibrations into a HIL environment. A block scheme of the proposed methodology describing the whole process, featured by two steps: offline and real-time, is shown in Figure 9. The virtual calibration described in this work, based on SIL technique, represents the off-line process in the upper side of the scheme, which could be suitably connected to the real-time HIL system.

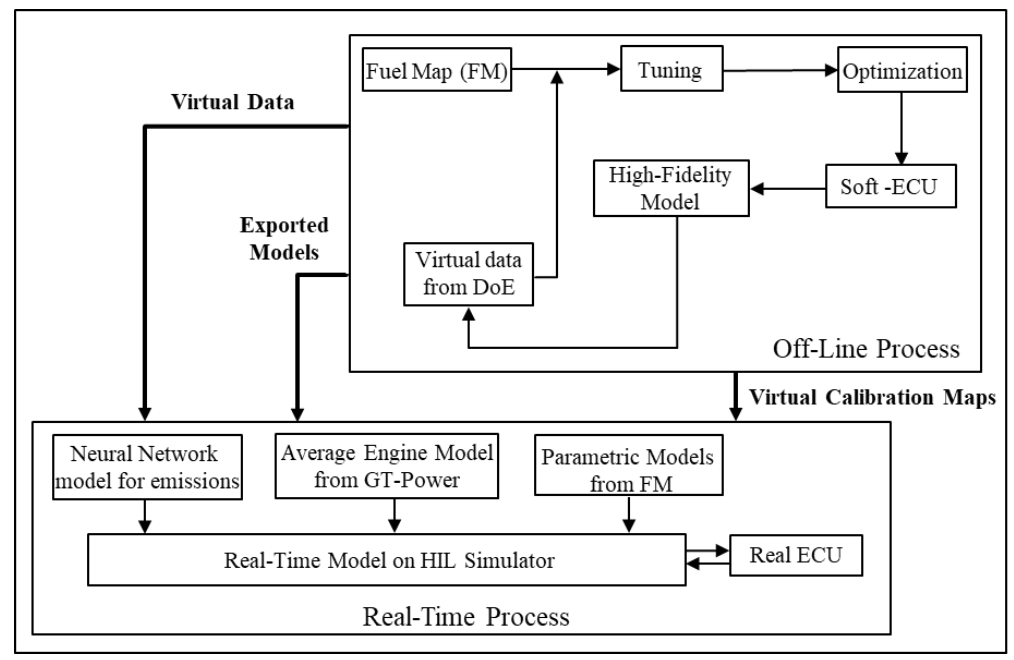

Figure 9. Block scheme of the whole process.

In this way, it is possible to consider external conditions in the virtual environment, not easily replicable in an engine test cell (because of operative conditions and costs), such as ageing effects on components and environment conditions. At this point, accordingly, machine learning techniques are even more suitable for classifying engine behaviour in different conditions compared to the standard operating points obtained from the only engine map from test-bench. 


\section{CONCLUSION}

This paper describes the virtual calibration process, also called software in the loop, a process that has the aim to reduce the time and costs of the traditional calibration process performed directly on the engine test bench. As a future activity, an extension to hardware in the loop testing can be possible starting from software in the loop method, by using real-time estimators starting from virtual data.

Modern engines require a high tests number both for calibration and experimental activity. The manual execution and without intelligent techniques of such tests involves the exponential increase of the time to market. This leads to the development of virtual calibrations, repeatable because they are not influenced by boundary conditions such as the external environment. A phenomenological model is used for application in a virtual diesel engine calibration. The methodology used can be described in a few points:

i. A model-based calibration toolbox, available in the Matlab software, allowed the generation of DOE, minimises measurement efforts by reducing the number of tests to be performed and the calibration time. Specifically, the DOE considered the interactions among the parameters that mainly influence the emissions (the injection start angle, the EGR fraction, the engine speed, the average effective pressure and the rail pressure).

ii. A model of the engine was built by a 1-D code; it was based on theoretical maps as reference.

iii. An optimisation model allowed to obtain new optimal calibration maps. The target was to reduce the pollutant emissions in a diesel engine, keeping good performance by imposing constrains on the torque.

iv. Good results have been carried out with the optimisation:

v. The polluting emissions of $\mathrm{NO}_{\mathrm{x}}$ and $\mathrm{CO}$ decreased by about $30 \%$ respect to the values obtained with the original engine map;

vi. The specific fuel consumption decreased by $17 \%$ respect to the standard engine map.

Therefore, the achieved results have validated the virtual calibration iterative process, giving appropriate results for each polluting species considered in particular $\mathrm{NO}_{\mathrm{x}}$ amount with a reduction of specific fuel consumption too. By improving the accuracy of the model and using the experimental engine map, a further improvement of the results should be expected. However, the concept of virtual engine calibration can only be as good as the model's ability to reproduce the complex physics of the engine in all model parts. Nevertheless, the demonstrated advantageous aspects of a model-based calibration method can be used in the engine development process to promote optimised energy conversion in the engine to reduce pollutants.

\section{ACKNOWLEDGEMENT}

The authors thank Dr Marco Amodeo for his useful contribution to the investigation.

\section{REFERENCES}

[1] Perez F, Moulin P, Del Mastro A. Vehicle simulation on an engine test bed. In: SIA International Conference, Diesel Engines - The low CO2 \& Emissions Reduction Challenge, pp. 1-8, 2008.

[2] Grasreiner S, Neumann J, Wensing M, Hasse C. Model-based virtual engine 
calibration with the help of phenomenological methods for spark-ignited engines. Applied Thermal Engineering 2017; 121:190-199.

[3] Turkson RF, Yan F, Ali MKA, Hu J. Artificial neural network applications in the calibration of spark-ignition engines: An overview. International Journal 2016; 1346-1359.

[4] Zaglauer S, Knoll U. Evolutionary Algorithms for the Automatic Calibration of Simulation Models for the Virtual Engine Application. In: IFAC Proceedings, pp. 177-181; 2012.

[5] Beccari A, Beccari S, Pipitone E. An analytical approach for the evaluation of the optimal combustion phase in spark ignition engines. Journal of Engineering for Gas Turbines and Power, 2010; 132(3): 032802.

[6] Pipitone E, Beccari S, Genchi G. Calibration of a knock prediction model for the combustion of gasoline-natural gas mixtures in spark ignition engines. Combustion Science and Technology, 2014; 187(5): 721-738.

[7] Teodosio L, Tufano D, Bozza F. Development of a virtual calibration methodology for a downsized SI engine by using advanced valve strategies. Energy Procedia, 2017; (126): 923-930.

[8] Tietze N. Model-based Calibration of Engine Control Units Using Gaussian Process Regression. Degree Thesis, Techischen Universitat Darmstadt, 2015.

[9] Vihar R, Baskovic UZ, Katrasnik T. Real-time capable virtual NOx sensor for diesel engines based on a two-zone thermodynamic model. IFP Energies nouvelles 2018; 73(11): 1-17.

[10] Castagne M, Bentolila Y, Chaudoye F. Comparison of engine calibration methods based on design of experiments (DoE). Oil \& Gas Science and Technology, 2008; (63): 563-582.

[11] Guerrier M, Cawsey P. The development of model based methodologies for gasoline IC engine calibration. SAE Technical Paper: 2004-01-1466, 2004.

[12] Schlosser A, Schönefelder C, Hendrikx M, Pischinger S, Sentis T. Automated ECU calibration - example: torque structure of gasoline engine. In: 5th Design of Experiment (DoE) in Engine Development System, Berlin, Germany, pp. 137150; 2009.

[13] Berger B, Rauscher F. Robust Gaussian process modelling for engine calibration. In: 7th Vienna International Conference on Mathematical Modelling, pp. 159$164 ; 2012$.

[14] Kruse T, Huber T, Ulmer H, Gutjahr T. New approaches for the data driven modelling of dynamic engine behaviour. In: $1^{\text {st }}$ International Conference on Powertrain Modelling Control, Bradford, U.K., pp. 1- 10; 2014.

[15] Rizzoni G. An improved model-based methodology for calibration of an alternative fueled engine. Degree Thesis, The Ohio State University, 2011.

[16] Rajesh A, Venkatesh J. Taguchi method and Pareto ANOVA: An approach for process parameters optimisation in micro-EDM drilling. International Journal of Scientific \& Engineering Research, 2014; 5(10): 38-42.

[17] Gokce M, Kozan R. Two-stage engine mapping for the calibration of carbon monoxide emission. Modern Applied Science 2009; 3(4): 30-36.

[18] Jiang S, Smith M H, Kitchen J. Development of an engine-in-the-loop vehicle simulation system in engine dynamometer test cell. SAE Technical Paper: 200901-1039, 2009.

[19] Yan Q, Williams J, Li J. Chassis control system development using simulation: software in the loop, rapid prototyping, and hardware in the loop. SAE paper: 
2002-01-1565, 2002.

[20] Demers S, Gopalakrishnan P, Kant L. A generic solution to software in the loop. Military Communications Conference, 2007.

[21] Grasreiner S. Combustion modelling for virtual SI engine calibration with the help of 0D/3D methods. Degree Thesis, Technical University Bergakademie Freiberg, 2012.

[22] Nola FD, Giardiello G, Gimelli A, Molteni A, Picariello R, Tornese D. Reduction of the experimental effort in engine calibration by using neural networks and 1D engine simulation. Energy Procedia 2018; :344-351.

[23] Bozza F, De Bellis V, Teodosio L. A numerical procedure for the calibration of a turbocharged spark-ignition variable valve actuation engine at part load. International Journal of Engine Research, 2016; 1-14.

[24] King J. A turbocharger unsteady performance model for the GT-Power internal combustion engine simulation. Degree Thesis, Purdue University, 2002.

[25] Shaohua Z, Guodong J, Tianfeng X. Design and analysis method for internal combustion engine mufflers based of GT-Power software. Degree Thesis, Huazhong University of Science and Technology, 2008.

[26] Morton T, Connors R, Maloney P, Sampson D. Model-based optimal calibration of a dual independent variable valve-timing engine, design of experiments (DOE). In: Der Motorenentwicklung, Expert Verlag, pp. 77-85; 2003.

[27] Fathy HK, Filipi ZS, Hagena J, Stein JL. Review of hardware-in-the-loop simulation and its prospects in the automotive area. In: Modelling and Simulation for Military Applications, 62280E, 2006. 